\title{
Endoscopie colique, coloscopie, colonoscopie, dépistage ou prévention : la monoculture ou le monothéisme est-il un événement porteur de risque ?
}

\section{Colon endoscopy, colonoscopy, screening or prevention: are monoculture or monotheism risk factors?}

\author{
D. Heresbach \\ (C) Springer-Verlag France 2010
}

L'endoscopie du côlon ou coloscopie est très présente dans les thèmes abordés dans les différentes réunions d'information, de formation, d'évaluation de la spécialité, et cette année encore, aussi bien aux journées de réflexion qu'au symposium de la SFED ou dans l'article original de ce numéro d'Acta Endoscopica, elle sera au premier plan. Certes, de nombreux arguments plaident pour cette mise en exergue, tant sur le plan quantitatif (1,3 million de coloscopies réalisées annuellement en France) que politique (efficacité et impact du dépistage sur les 37400 cancers colorectaux et 16900 décès annuels) ou financier. Néanmoins, la monoculture n'a jamais été source de progrès, surtout si cette exploration doit vivre durant les années à venir un changement dans sa pratique diagnostique (place de la coloscopie virtuelle ou de la vidéocapsule colique) et thérapeutique (place de la dissection sousmuqueuse ou du Notes). Bien que ces changements soient initiés, encouragés, accompagnés, suivis ou méprisés par les gastroentérologues, cette focalisation comporte le risque de se voir imposer des changements non pas tant par les tutelles que par la vox populi. En effet, l'épisode du surplus de vaccins contre la grippe H1N1, au-delà de la possibilité d'anticiper ou pas le risque pandémique ou sa gravité, et au-delà du principe de précaution, a montré que les « consommateurs » et non les «malades », puisqu'il s'agit bien de prévention et non de soins, désiraient rester maîtres de leur choix, avec une méfiance vis-à-vis des conseillers, d'autant plus que ces derniers étaient promoteurs de la méthode.

Cette gestion devrait nous alerter pour les futures politiques de dépistage et montre que, sauf à adopter une politique fortement incitative ou coercitive, le consommateur sera toujours méfiant vis-à-vis d'une politique ou d'un discours monolithique ou monoculturel. Certes, syndicats, sociétés savantes, associations professionnelles et ministères défendent et expliquent « mieux et plus » les bien-fondés de telle ou telle politique sur des arguments pas toujours objectifs, scientifiques ou impartiaux. Le deuxième Plan cancer vise à renseigner les raisons de l'inégalité d'accès au dépistage et à mettre en place les solutions pour y remédier ; évidemment, tous rêvent d'un futur et d'un avenir meilleurs, et d'être en bonne santé sous nos latitudes. Mais les aspirations de nos concitoyens ne seraient-elles pas tout simplement de choisir le taux ou la part d'investissement pour améliorer le quotidien et celui à investir dans l'avenir. Une information loyale et éclairée n'inclue-t-elle pas un libre choix des modalités de dépistage (et naturellement leurs conséquences) ? Devronsnous attendre que des « class actions » ou « associations de faux négatifs » se créent pour voir surgir une inflation des événements porteurs de risque ? Ce " jour d'après », nous n'aurons pas l'excuse de la méconnaissance ou de l'absence de données dans la littérature.

D. Heresbach $(\triangle)$

Service des maladies de l'appareil digestif,

CHU de Pontchaillou, 2, rue Henri-Le-Guilloux,

F-35033 Rennes cedex 9, France

e-mail : denis.heresbach@chu-rennes.fr 